\title{
Trapezoid type inequalities for generalized Riemann-Liouville fractional integrals of functions with bounded variation
}

\author{
Silvestru Sever Dragomir \\ Mathematics, College of Engineering \& Science \\ Victoria University, PO Box 14428 \\ Melbourne City, MC 8001, Australia \\ email: sever.dragomir@vu.edu.au \\ URL: http://rgmia.org/dragomir \\ DST-NRF Centre of Excellence \\ in the Mathematical and Statistical Sciences, \\ School of Computer Science \& Applied Mathematics, \\ University of the Witwatersrand, Private Bag 3, \\ Johannesburg 2050, South Africa
}

\begin{abstract}
In this paper we establish some trapezoid type inequalities for the Riemann-Liouville fractional integrals of functions of bounded variation and of Hölder continuous functions. Applications for the $\mathrm{g}$ mean of two numbers are provided as well. Some particular cases for Hadamard fractional integrals are also provided.
\end{abstract}

\section{Introduction}

Let $(a, b)$ with $-\infty \leq a<b \leq \infty$ be a finite or infinite interval of the real line $\mathbb{R}$ and $\alpha$ a complex number with $\operatorname{Re}(\alpha)>0$. Also let $g$ be a strictly increasing function on $(a, b)$, having a continuous derivative $g^{\prime}$ on $(a, b)$. Following [18,

2010 Mathematics Subject Classification: 26D15, 26D10, 26D07, 26A33

Key words and phrases: Riemann-Liouville fractional integrals, functions of bounded variation, Lipshitzian functions, trapezoid type inequalities 
p. 100], we introduce the generalized left- and right-sided Riemann-Liouville fractional integrals of a function $f$ with respect to another function $g$ on $[a, b]$ by

$$
I_{a+, g}^{\alpha} f(x):=\frac{1}{\Gamma(\alpha)} \int_{a}^{x} \frac{g^{\prime}(t) f(t) d t}{[g(x)-g(t)]^{1-\alpha}}, a<x \leq b
$$

and

$$
\mathrm{I}_{\mathrm{b}-, \mathrm{g}}^{\alpha} \mathrm{f}(\mathrm{x}):=\frac{1}{\Gamma(\alpha)} \int_{x}^{\mathrm{b}} \frac{g^{\prime}(\mathrm{t}) \mathrm{f}(\mathrm{t}) \mathrm{dt}}{[\mathrm{g}(\mathrm{t})-\mathrm{g}(\mathrm{x})]^{1-\alpha}}, \quad \mathrm{a} \leq \mathrm{x}<\mathrm{b} .
$$

For $\mathrm{g}(\mathrm{t})=\mathrm{t}$ we have the classical Riemann-Liouville fractional integrals

$$
J_{a+}^{\alpha} f(x):=\frac{1}{\Gamma(\alpha)} \int_{a}^{x} \frac{f(t) d t}{(x-t)^{1-\alpha}}, a<x \leq b
$$

and

$$
J_{b-}^{\alpha} f(x):=\frac{1}{\Gamma(\alpha)} \int_{x}^{b} \frac{f(t) d t}{(t-x)^{1-\alpha}}, a \leq x<b,
$$

while for the logarithmic function $\mathrm{g}(\mathrm{t})=\ln \mathrm{t}$ we have the Hadamard fractional integrals [18, p. 111]

$$
H_{a+}^{\alpha} f(x):=\frac{1}{\Gamma(\alpha)} \int_{a}^{x}\left[\ln \left(\frac{x}{t}\right)\right]^{\alpha-1} \frac{f(t) d t}{t}, 0 \leq a<x \leq b
$$

and

$$
H_{b-}^{\alpha} f(x):=\frac{1}{\Gamma(\alpha)} \int_{x}^{b}\left[\ln \left(\frac{t}{x}\right)\right]^{\alpha-1} \frac{f(t) d t}{t}, 0 \leq a<x<b .
$$

One can consider the function $\mathrm{g}(\mathrm{t})=-\mathrm{t}^{-1}$ and define the "Harmonic fractional integrals" by

$$
R_{a+}^{\alpha} f(x):=\frac{x^{1-\alpha}}{\Gamma(\alpha)} \int_{a}^{x} \frac{f(t) d t}{(x-t)^{1-\alpha} t^{\alpha+1}}, 0 \leq a<x \leq b
$$

and

$$
R_{b-}^{\alpha} f(x):=\frac{x^{1-\alpha}}{\Gamma(\alpha)} \int_{x}^{b} \frac{f(t) d t}{(t-x)^{1-\alpha} t^{\alpha+1}}, 0 \leq a<x<b .
$$

Also, for $\mathrm{g}(\mathrm{t})=\exp (\beta \mathrm{t}), \beta>0$, we can consider the " $\beta$-Exponential fractional integrals"

$$
E_{a+, \beta}^{\alpha} f(x):=\frac{\beta}{\Gamma(\alpha)} \int_{a}^{x} \frac{\exp (\beta t) f(t) d t}{[\exp (\beta x)-\exp (\beta t)]^{1-\alpha}}, a<x \leq b
$$


and

$$
E_{b-, \beta}^{\alpha} f(x):=\frac{\beta}{\Gamma(\alpha)} \int_{x}^{b} \frac{\exp (\beta t) f(t) d t}{[\exp (\beta t)-\exp (\beta x)]^{1-\alpha}}, a \leq x<b .
$$

In the recent paper [14] we obtained the following Ostrowski type inequalities for functions of bounded variation:

Theorem 1 Let $\mathrm{f}:[\mathrm{a}, \mathrm{b}] \rightarrow \mathbb{C}$ be a function of bounded variation on $[\mathrm{a}, \mathrm{b}]$ and $\mathrm{g}$ be a strictly increasing function on $(\mathrm{a}, \mathrm{b})$, having a continuous derivative $\mathrm{g}^{\prime}$ on $(\mathrm{a}, \mathrm{b})$. For any $\mathrm{x} \in(\mathrm{a}, \mathrm{b})$ we have the inequalities

$$
\begin{aligned}
& \left|I_{a+, g}^{\alpha} f(x)+I_{b-, g}^{\alpha} f(x)-\frac{1}{\Gamma(\alpha+1)}\left([g(x)-g(a)]^{\alpha}+[g(b)-g(x)]^{\alpha}\right) f(x)\right| \\
& \leq \frac{1}{\Gamma(\alpha)}\left[\int_{a}^{x} \frac{g^{\prime}(t) \bigvee_{t}^{x}(f) d t}{[g(x)-g(t)]^{1-\alpha}}+\int_{x}^{b} \frac{g^{\prime}(t) \bigvee_{x}^{t}(f) d t}{[g(t)-g(x)]^{1-\alpha}}\right] \\
& \leq \frac{1}{\Gamma(\alpha+1)}\left[[g(x)-g(a)]^{\alpha} \bigvee_{a}^{x}(f)+[g(b)-g(x)]^{\alpha} \bigvee_{x}^{b}(f)\right] \\
& \leq \frac{1}{\Gamma(\alpha+1)}\left\{\begin{array}{l}
{\left[\frac{1}{2}(g(b)-g(a))+\left|g(x)-\frac{g(a)+g(b)}{2}\right|\right]^{\alpha} \bigvee_{a}^{b}(f) ;} \\
\left((g(x)-g(a))^{\alpha p}+(g(b)-g(x))^{\alpha p}\right)^{1 / p}\left(\left(\bigvee_{a}^{x}(f)\right)^{q}+\left(\bigvee_{x}^{b}(f)\right)^{q}\right)^{1 / q} \\
\text { with p, q >1, } \frac{1}{p}+\frac{1}{q}=1 ; \\
\left((g(x)-g(a))^{\alpha}+(g(b)-g(x))^{\alpha}\right)\left[\frac{1}{2} \bigvee_{a}^{b}(f)+\frac{1}{2}\left|\bigvee_{a}^{x}(f)-\bigvee_{x}^{b}(f)\right|\right],
\end{array}\right.
\end{aligned}
$$

and

$$
\begin{aligned}
& \left|I_{x-, g}^{\alpha} f(a)+I_{x+, g}^{\alpha} f(b)-\frac{1}{\Gamma(\alpha+1)}\left([g(x)-g(a)]^{\alpha}+[g(b)-g(x)]^{\alpha}\right) f(x)\right| \\
& \leq \frac{1}{\Gamma(\alpha)}\left[\int_{a}^{x} \frac{g^{\prime}(t) \bigvee_{t}^{x}(f) d t}{[g(t)-g(a)]^{1-\alpha}}+\int_{x}^{b} \frac{g^{\prime}(t) \bigvee_{x}^{t}(f) d t}{[g(b)-g(t)]^{1-\alpha}}\right] \\
& \leq \frac{1}{\Gamma(\alpha+1)}\left[[g(x)-g(a)]^{\alpha} \bigvee_{a}^{x}(f)+[g(b)-g(x)]^{\alpha} \bigvee_{x}^{b}(f)\right] \\
& \leq \frac{1}{\Gamma(\alpha+1)}\left\{\begin{array}{l}
{\left[\frac{1}{2}(g(b)-g(a))+\left|g(x)-\frac{g(a)+g(b)}{2}\right|\right]^{\alpha} \bigvee_{a}^{b}(f) ;} \\
\left((g(x)-g(a))^{\alpha p}+(g(b)-g(x))^{\alpha p}\right)^{1 / p}\left(\left(\bigvee_{a}^{x}(f)\right)^{q}+\left(\bigvee_{x}^{b}(f)\right)^{q}\right)^{1 / q} \\
w i t h p, q>1, \frac{1}{p}+\frac{1}{q}=1 ; \\
\left((g(x)-g(a))^{\alpha}+(g(b)-g(x))^{\alpha}\right)\left[\frac{1}{2} \bigvee_{a}^{b}(f)+\frac{1}{2}\left|\bigvee_{a}^{x}(f)-\bigvee_{x}^{b}(f)\right|\right] .
\end{array}\right.
\end{aligned}
$$


If $g$ is a function which maps an interval I of the real line to the real numbers, and is both continuous and injective then we can define the g-mean of two numbers $\mathrm{a}, \mathrm{b} \in \mathrm{I}$ as

$$
M_{g}(a, b):=g^{-1}\left(\frac{g(a)+g(b)}{2}\right) .
$$

If $I=\mathbb{R}$ and $g(t)=t$ is the identity function, then $M_{g}(a, b)=A(a, b):=$ $\frac{\mathrm{a}+\mathrm{b}}{2}$, the arithmetic mean. If $\mathrm{I}=(0, \infty)$ and $\mathrm{g}(\mathrm{t})=\ln \mathrm{t}$, then $\mathrm{M}_{\mathrm{g}}(\mathrm{a}, \mathrm{b})=$ $\mathrm{G}(\mathrm{a}, \mathrm{b}):=\sqrt{\mathrm{ab}}$, the geometric mean. If $\mathrm{I}=(0, \infty)$ and $\mathrm{g}(\mathrm{t})=\frac{1}{\mathrm{t}}$, then $M_{g}(a, b)=H(a, b):=\frac{2 a b}{a+b}$, the harmonic mean. If $I=(0, \infty)$ and $g(t)=t^{p}$, $p \neq 0$, then $M_{g}(a, b)=M_{p}(a, b):=\left(\frac{a^{p}+b^{p}}{2}\right)^{1 / p}$, the power mean with exponent $\mathrm{p}$. Finally, if $\mathrm{I}=\mathbb{R}$ and $\mathrm{g}(\mathrm{t})=\exp \mathrm{t}$, then

$$
M_{g}(a, b)=\operatorname{LME}(a, b):=\ln \left(\frac{\exp a+\exp b}{2}\right),
$$

the LogMeanExp function.

The following particular case for g-mean is of interest [14].

Corollary 1 With the assumptions of Theorem 1 we have

$$
\begin{gathered}
\left|I_{a+, g}^{\alpha} f\left(M_{g}(a, b)\right)+I_{b-, g}^{\alpha} f\left(M_{g}(a, b)\right)-\frac{[g(b)-g(a)]^{\alpha}}{2^{\alpha-1} \Gamma(\alpha+1)} f\left(M_{g}(a, b)\right)\right| \\
\leq \frac{1}{\Gamma(\alpha)}\left[\int_{a}^{M_{g}(a, b)} \frac{g^{\prime}(t) \bigvee_{t}^{M_{g}(a, b)}(f) d t}{\left[g\left(M_{g}(a, b)\right)-g(t)\right]^{1-\alpha}}+\int_{M_{g}(a, b)}^{b} \frac{g^{\prime}(t) \bigvee_{M_{g}(a, b)}^{t}(f) d t}{\left[g(t)-g\left(M_{g}(a, b)\right)\right]^{1-\alpha}}\right] \\
\leq \frac{1}{2^{\alpha} \Gamma(\alpha+1)}(g(b)-g(a))^{\alpha} \bigvee_{a}^{b}(f)
\end{gathered}
$$

and

$$
\begin{aligned}
& \left|I_{M_{g}(a, b)-, g}^{\alpha} f(a)+I_{M_{g}(a, b)+, g}^{\alpha} f(b)-\frac{[g(b)-g(a)]^{\alpha}}{2^{\alpha-1} \Gamma(\alpha+1)} f\left(M_{g}(a, b)\right)\right| \\
& \leq \frac{1}{\Gamma(\alpha)}\left[\int_{a}^{M_{g}(a, b)} \frac{g^{\prime}(t) \bigvee_{t}^{M_{g}(a, b)}(f) d t}{[g(t)-g(a)]^{1-\alpha}}+\int_{M_{g}(a, b)}^{b} \frac{g^{\prime}(t) \bigvee_{x}^{t}(f) d t}{[g(b)-g(t)]^{1-\alpha}}\right] \\
& \leq \frac{1}{2^{\alpha} \Gamma(\alpha+1)}(g(b)-g(a))^{\alpha} \bigvee_{a}^{b}(f) .
\end{aligned}
$$


Remark 1 If we take in Theorem $1 \mathrm{x}=\frac{\mathrm{a}+\mathrm{b}}{2}$, then we obtain similar mid-point inequalities, however the details are not presented here. Some applications for the Hadamard fractional integrals are also provided in [14].

For several Ostrowski type inequalities for Riemann-Liouville fractional integrals see [1]-[5], [16]-[27] and the references therein.

Motivated by the above results, in this paper we establish some trapezoid type inequalities for the generalized Riemann-Liouville fractional integrals of functions of bounded variation and of Hölder continuous functions. Applications for the g-mean of two numbers are provided as well. Some particular cases for Hadamard fractional integrals are also provided.

\section{Some identities}

We have:

Lemma 1 Let $\mathrm{f}:[\mathrm{a}, \mathrm{b}] \rightarrow \mathbb{C}$ be Lebesgue integrable on $[\mathrm{a}, \mathrm{b}], \mathrm{g}$ be a strictly increasing function on $(\mathrm{a}, \mathrm{b})$, having a continuous derivative $\mathrm{g}^{\prime}$ on $(\mathrm{a}, \mathrm{b})$ and $\lambda, \mu$ some complex parameters:

(i) For any $\mathrm{x} \in(\mathrm{a}, \mathrm{b})$ we have the representation

$$
\begin{array}{r}
I_{a+, g}^{\alpha} f(x)+I_{b-, g}^{\alpha} f(x)=\frac{1}{\Gamma(\alpha+1)}\left(\lambda[g(x)-g(a)]^{\alpha}+\mu[g(b)-g(x)]^{\alpha}\right) \\
+\frac{1}{\Gamma(\alpha)}\left[\int_{a}^{x} \frac{g^{\prime}(t)[f(t)-\lambda] d t}{[g(x)-g(t)]^{1-\alpha}}+\int_{x}^{b} \frac{g^{\prime}(t)[f(t)-\mu] d t}{[g(t)-g(x)]^{1-\alpha}}\right]
\end{array}
$$

and

$$
\begin{aligned}
& I_{x-, g}^{\alpha} f(a)+I_{x+, g}^{\alpha} f(b)=\frac{1}{\Gamma(\alpha+1)}\left(\lambda[g(x)-g(a)]^{\alpha}+\mu[g(b)-g(x)]^{\alpha}\right) \\
&+\frac{1}{\Gamma(\alpha)}\left[\int_{a}^{x} \frac{g^{\prime}(t)[f(t)-\lambda] d t}{[g(t)-g(a)]^{1-\alpha}}+\int_{x}^{b} \frac{g^{\prime}(t)[f(t)-\mu] d t}{[g(b)-g(t)]^{1-\alpha}}\right] .
\end{aligned}
$$

(ii) We have

$$
\begin{aligned}
& \frac{I_{b-, g}^{\alpha} f(a)+I_{a+, g}^{\alpha} f(b)}{2}=\frac{1}{\Gamma(\alpha+1)}[g(b)-g(a)]^{\alpha} \frac{\lambda+\mu}{2} \\
&+\frac{1}{2 \Gamma(\alpha)}\left[\int_{a}^{b} \frac{g^{\prime}(t)[f(t)-\lambda] d t}{[g(b)-g(t)]^{1-\alpha}}+\int_{a}^{b} \frac{g^{\prime}(t)[f(t)-\mu] d t}{[g(t)-g(a)]^{1-\alpha}}\right] .
\end{aligned}
$$


Proof. (i) We observe that

$$
\begin{aligned}
& \frac{1}{\Gamma(\alpha)} \int_{a}^{x} \frac{g^{\prime}(t)[f(t)-\lambda] d t}{[g(x)-g(t)]^{1-\alpha}} \\
& =I_{a+, g}^{\alpha} f(x)-\lambda \frac{1}{\Gamma(\alpha)} \int_{a}^{x} \frac{g^{\prime}(t) d t}{[g(x)-g(t)]^{1-\alpha}} \\
& =I_{a+, g}^{\alpha} f(x)-\frac{[g(x)-g(a)]^{\alpha}}{\alpha \Gamma(\alpha)} \lambda=I_{a+, g}^{\alpha} f(x)-\frac{[g(x)-g(a)]^{\alpha}}{\Gamma(\alpha+1)} \lambda
\end{aligned}
$$

for $\mathrm{a}<\mathrm{x} \leq \mathrm{b}$ and, similarly,

$$
\frac{1}{\Gamma(\alpha)} \int_{x}^{b} \frac{g^{\prime}(t)[f(t)-\mu] d t}{[g(t)-g(x)]^{1-\alpha}}=I_{b-, g}^{\alpha} f(x)-\frac{[g(b)-g(x)]^{\alpha}}{\Gamma(\alpha+1)} \mu
$$

for $a \leq x<b$.

If $x \in(a, b)$, then by adding the equalities (4) and (5) we get the representation (1).

By the definition of fractional integrals we have

$$
I_{x+, g}^{\alpha} f(b):=\frac{1}{\Gamma(\alpha)} \int_{x}^{b} \frac{g^{\prime}(t) f(t) d t}{[g(b)-g(t)]^{1-\alpha}}, a \leq x<b
$$

and

$$
I_{x-, g}^{\alpha} f(a):=\frac{1}{\Gamma(\alpha)} \int_{a}^{x} \frac{g^{\prime}(t) f(t) d t}{[g(t)-g(a)]^{1-\alpha}}, a<x \leq b
$$

Then

$$
\frac{1}{\Gamma(\alpha)} \int_{x}^{b} \frac{g^{\prime}(t)[f(t)-\lambda] d t}{[g(b)-g(t)]^{1-\alpha}}=I_{x+, g}^{\alpha} f(b)-\frac{[g(b)-g(x)]^{\alpha}}{\Gamma(\alpha+1)} \lambda
$$

for $a \leq x<b$ and

$$
\frac{1}{\Gamma(\alpha)} \int_{a}^{x} \frac{g^{\prime}(t)[f(t)-\mu] d t}{[g(t)-g(a)]^{1-\alpha}}=I_{x-, g}^{\alpha} f(a)-\frac{[g(x)-g(a)]^{\alpha}}{\Gamma(\alpha+1)} \mu
$$

for $a<x \leq b$.

If $x \in(a, b)$, then by adding the equalities (6) and (7) we get the representation (1).

If we take $x=b$ in (4) we get

$$
\frac{1}{\Gamma(\alpha)} \int_{a}^{b} \frac{g^{\prime}(t)[f(t)-\lambda] d t}{[g(b)-g(t)]^{1-\alpha}}=I_{a+, g}^{\alpha} f(b)-\frac{[g(b)-g(a)]^{\alpha}}{\Gamma(\alpha+1)} \lambda
$$


while from $x=a$ in (5) we get

$$
\frac{1}{\Gamma(\alpha)} \int_{a}^{b} \frac{g^{\prime}(t)[f(t)-\mu] d t}{[g(t)-g(a)]^{1-\alpha}}=I_{b-, g}^{\alpha} f(a)-\frac{[g(b)-g(a)]^{\alpha}}{\Gamma(\alpha+1)} \mu .
$$

If we add (8) with (9) and divide by 2 we get (3).

Remark 2 If we take in (1) and (2) $x=M_{g}(a, b)=g^{-1}\left(\frac{g(a)+g(b)}{2}\right)$, then we get

$$
\begin{gathered}
I_{a+, g}^{\alpha} f\left(M_{g}(a, b)\right)+I_{b-, g}^{\alpha} f\left(M_{g}(a, b)\right) \\
=\frac{1}{2^{\alpha-1} \Gamma(\alpha+1)}[g(b)-g(a)]^{\alpha}\left(\frac{\lambda+\mu}{2}\right) \\
+\frac{1}{\Gamma(\alpha)}\left[\int_{a}^{M_{g}(a, b)} \frac{g^{\prime}(t)[f(t)-\lambda] d t}{\left[g\left(M_{g}(a, b)\right)-g(t)\right]^{1-\alpha}}+\int_{M_{g}(a, b)}^{b} \frac{g^{\prime}(t)[f(t)-\mu] d t}{\left[g(t)-g\left(M_{g}(a, b)\right)\right]^{1-\alpha}}\right]
\end{gathered}
$$

and

$$
\begin{aligned}
& I_{M_{g}(a, b)-, g}^{\alpha} f(a)+I_{M_{g}(a, b)+, g}^{\alpha} f(b)=\frac{1}{2^{\alpha-1} \Gamma(\alpha+1)}[g(b)-g(a)]^{\alpha}\left(\frac{\lambda+\mu}{2}\right) \\
& \quad+\frac{1}{\Gamma(\alpha)}\left[\int_{a}^{M_{g}(a, b)} \frac{g^{\prime}(t)[f(t)-\lambda] d t}{[g(t)-g(a)]^{1-\alpha}}+\int_{M_{g}(a, b)}^{b} \frac{g^{\prime}(t)[f(t)-\mu] d t}{[g(b)-g(t)]^{1-\alpha}}\right] .
\end{aligned}
$$

The above lemma provides various identities of interest by taking particular values for the parameters $\lambda$ and $\mu$, out of which we give only a few:

Corollary 2 With the assumptions of Lemma 1 we have:

(i) For any $x \in(a, b)$,

$$
\begin{aligned}
I_{a+, g}^{\alpha} f(x) & +I_{b-, g}^{\alpha} f(x)=\frac{1}{\Gamma(\alpha+1)}\left([g(x)-g(a)]^{\alpha}+[g(b)-g(x)]^{\alpha}\right) f(x) \\
+ & \frac{1}{\Gamma(\alpha)}\left[\int_{a}^{x} \frac{g^{\prime}(t)[f(t)-f(x)] d t}{[g(x)-g(t)]^{1-\alpha}}+\int_{x}^{b} \frac{g^{\prime}(t)[f(t)-f(x)] d t}{[g(t)-g(x)]^{1-\alpha}}\right]
\end{aligned}
$$

and

$$
I_{x-, g}^{\alpha} f(a)+I_{x+, g}^{\alpha} f(b)=\frac{1}{\Gamma(\alpha+1)}\left([g(x)-g(a)]^{\alpha}+[g(b)-g(x)]^{\alpha}\right) f(x)
$$




$$
+\frac{1}{\Gamma(\alpha)}\left[\int_{a}^{x} \frac{g^{\prime}(t)[f(t)-f(x)] d t}{[g(t)-g(a)]^{1-\alpha}}+\int_{x}^{b} \frac{g^{\prime}(t)[f(t)-f(x)] d t}{[g(b)-g(t)]^{1-\alpha}}\right] .
$$

(ii) For any $x \in[a, b]$,

$$
\begin{aligned}
& \frac{I_{b-, g}^{\alpha} f(a)+I_{a+, g}^{\alpha} f(b)}{2}=\frac{1}{\Gamma(\alpha+1)}[g(b)-g(a)]^{\alpha} f(x) \\
& \quad+\frac{1}{2 \Gamma(\alpha)}\left[\int_{a}^{b} \frac{g^{\prime}(t)[f(t)-f(x)] d t}{[g(b)-g(t)]^{1-\alpha}}+\int_{a}^{b} \frac{g^{\prime}(t)[f(t)-f(x)] d t}{[g(t)-g(a)]^{1-\alpha}}\right] .
\end{aligned}
$$

The proof is obvious by taking $\lambda=\mu=f(x)$ in Lemma 1. These identities were obtained in [14]. If we take in (10)-(12) $x=M_{g}(a, b)=g^{-1}\left(\frac{g(a)+g(b)}{2}\right)$, then we get the corresponding identities were obtained in [14].

Corollary 3 With the assumptions of Lemma 1 we have:

$$
\begin{aligned}
I_{a+, g}^{\alpha} f(x) & +I_{b-, g}^{\alpha} f(x) \\
& =\frac{1}{\Gamma(\alpha+1)}\left([g(x)-g(a)]^{\alpha} f(a)+[g(b)-g(x)]^{\alpha} f(b)\right) \\
& +\frac{1}{\Gamma(\alpha)}\left[\int_{a}^{x} \frac{g^{\prime}(t)[f(t)-f(a)] d t}{[g(x)-g(t)]^{1-\alpha}}+\int_{x}^{b} \frac{g^{\prime}(t)[f(t)-f(b)] d t}{[g(t)-g(x)]^{1-\alpha}}\right]
\end{aligned}
$$

and

$$
\begin{aligned}
I_{x-, g}^{\alpha} f(a) & +I_{x+, g}^{\alpha} f(b) \\
& =\frac{1}{\Gamma(\alpha+1)}\left([g(x)-g(a)]^{\alpha} f(a)+[g(b)-g(x)]^{\alpha} f(b)\right) \\
+ & \frac{1}{\Gamma(\alpha)}\left[\int_{a}^{x} \frac{g^{\prime}(t)[f(t)-f(a)] d t}{[g(t)-g(a)]^{1-\alpha}}+\int_{x}^{b} \frac{g^{\prime}(t)[f(t)-f(b)] d t}{[g(b)-g(t)]^{1-\alpha}}\right],
\end{aligned}
$$

for any $\mathrm{x} \in(\mathrm{a}, \mathrm{b})$

(ii) We also have

$$
\begin{aligned}
& \frac{I_{b-, g}^{\alpha} f(a)+I_{a+, g}^{\alpha} f(b)}{2}=\frac{1}{\Gamma(\alpha+1)}[g(b)-g(a)]^{\alpha} \frac{f(b)+f(a)}{2} \\
& \quad+\frac{1}{2 \Gamma(\alpha)}\left[\int_{a}^{b} \frac{g^{\prime}(t)[f(t)-f(b)] d t}{[g(b)-g(t)]^{1-\alpha}}+\int_{a}^{b} \frac{g^{\prime}(t)[f(t)-f(a)] d t}{[g(t)-g(a)]^{1-\alpha}}\right] .
\end{aligned}
$$


The proof of (13) and (14) are obvious by taking $\lambda=f(a), \mu=f(b)$ in Lemma 1 . The proof of (15) follows by Lemma 1 on taking $\lambda=f(b)$ and $\mu=f(a)$.

Remark 3 If we take in (13) and (14) $x=M_{g}(a, b)=g^{-1}\left(\frac{g(a)+g(b)}{2}\right)$, then we get

$$
\begin{gathered}
I_{a+, g}^{\alpha} f\left(M_{g}(a, b)\right)+I_{b-, g}^{\alpha} f\left(M_{g}(a, b)\right) \\
=\frac{1}{2^{\alpha-1} \Gamma(\alpha+1)}[g(b)-g(a)]^{\alpha}\left(\frac{f(a)+f(b)}{2}\right) \\
+\frac{1}{\Gamma(\alpha)}\left[\int_{a}^{M_{g}(a, b)} \frac{g^{\prime}(t)[f(t)-f(a)] d t}{\left[g\left(M_{g}(a, b)\right)-g(t)\right]^{1-\alpha}}+\int_{M_{g}(a, b)}^{b} \frac{g^{\prime}(t)[f(t)-f(b)] d t}{\left[g(t)-g\left(M_{g}(a, b)\right)\right]^{1-\alpha}}\right]
\end{gathered}
$$

and

$$
\begin{aligned}
& I_{M_{g}(a, b)-, g}^{\alpha} f(a)+I_{M_{g}(a, b)+, g}^{\alpha} f(b) \\
& =\frac{1}{2^{\alpha-1} \Gamma(\alpha+1)}[g(b)-g(a)]^{\alpha}\left(\frac{f(a)+f(b)}{2}\right) \\
& +\frac{1}{\Gamma(\alpha)}\left[\int_{a}^{M_{g}(a, b)} \frac{g^{\prime}(t)[f(t)-f(a)] d t}{[g(t)-g(a)]^{1-\alpha}}+\int_{M_{g}(a, b)}^{b} \frac{g^{\prime}(t)[f(t)-f(b)] d t}{[g(b)-g(t)]^{1-\alpha}}\right] .
\end{aligned}
$$

\section{Inequalities for bounded functions}

Now, for $\phi, \Phi \in \mathbb{C}$ and $[a, b]$ an interval of real numbers, define the sets of complex-valued functions, see for instance [15]

$\overline{\mathrm{U}}_{[\mathrm{a}, \mathrm{b}]}(\phi, \Phi)$

$:=\{\mathrm{f}:[\mathrm{a}, \mathrm{b}] \rightarrow \mathbb{C} \mid \operatorname{Re}[(\Phi-\mathrm{f}(\mathrm{t}))(\overline{\mathrm{f}(\mathrm{t})}-\bar{\phi})] \geq 0$ for almost every $\mathrm{t} \in[\mathrm{a}, \mathrm{b}]\}$

and

$\bar{\Delta}_{[a, b]}(\phi, \Phi):=\left\{f:[a, b] \rightarrow \mathbb{C}\left|f(t)-\frac{\phi+\Phi}{2}\right| \leq \frac{1}{2}|\Phi-\phi|\right.$ for a.e. $\left.t \in[a, b]\right\}$.

The following representation result may be stated.

Proposition 1 For any $\phi, \Phi \in \mathbb{C}, \phi \neq \Phi$, we have that $\overline{\mathrm{U}}_{[\mathrm{a}, \mathrm{b}]}(\phi, \Phi)$ and $\bar{\Delta}_{[\mathrm{a}, \mathrm{b}]}(\phi, \Phi)$ are nonempty, convex and closed sets and

$$
\overline{\mathrm{U}}_{[\mathrm{a}, \mathrm{b}]}(\phi, \Phi)=\bar{\Delta}_{[\mathrm{a}, \mathrm{b}]}(\phi, \Phi) \text {. }
$$


Proof. We observe that for any $z \in \mathbb{C}$ we have the equivalence

$$
\left|z-\frac{\phi+\Phi}{2}\right| \leq \frac{1}{2}|\Phi-\phi|
$$

if and only if

$$
\operatorname{Re}[(\Phi-\mathrm{z})(\overline{\mathrm{z}}-\phi)] \geq 0 .
$$

This follows by the equality

$$
\frac{1}{4}|\Phi-\phi|^{2}-\left|z-\frac{\phi+\Phi}{2}\right|^{2}=\operatorname{Re}[(\Phi-\mathrm{z})(\overline{\mathrm{z}}-\phi)]
$$

that holds for any $z \in \mathbb{C}$.

The equality (16) is thus a simple consequence of this fact.

On making use of the complex numbers field properties we can also state that:

Corollary 4 For any $\phi, \Phi \in \mathbb{C}, \phi \neq \Phi$, we have that

$$
\begin{aligned}
\overline{\mathrm{U}}_{[\mathrm{a}, \mathrm{b}]}(\phi, \Phi)= & \{\mathrm{f}:[\mathrm{a}, \mathrm{b}] \rightarrow \mathbb{C} \mid(\operatorname{Re} \Phi-\operatorname{Ref}(\mathrm{t}))(\operatorname{Ref}(\mathrm{t})-\operatorname{Re} \phi) \\
& +(\operatorname{Im} \Phi-\operatorname{Imf}(\mathrm{t}))(\operatorname{Imf}(\mathrm{t})-\operatorname{Im} \phi) \geq 0 \text { for a.e. } \mathrm{t} \in[\mathrm{a}, \mathrm{b}]\} .
\end{aligned}
$$

Now, if we assume that $\operatorname{Re}(\Phi) \geq \operatorname{Re}(\phi)$ and $\operatorname{Im}(\Phi) \geq \operatorname{Im}(\phi)$, then we can define the following set of functions as well:

$$
\begin{aligned}
\bar{S}_{[\mathrm{a}, \mathrm{b}]}(\phi, \Phi) & :=\{\mathrm{f}:[\mathrm{a}, \mathrm{b}] \rightarrow \mathbb{C} \mid \operatorname{Re}(\Phi) \geq \operatorname{Ref}(\mathrm{t}) \geq \operatorname{Re}(\phi) \\
& \text { and } \operatorname{Im}(\Phi) \geq \operatorname{Imf}(\mathrm{t}) \geq \operatorname{Im}(\phi) \text { for a.e. } \mathrm{t} \in[\mathrm{a}, \mathrm{b}]\} .
\end{aligned}
$$

One can easily observe that $\overline{\mathrm{S}}_{[\mathrm{a}, \mathrm{b}]}(\phi, \Phi)$ is closed, convex and

$$
\emptyset \neq \overline{\mathrm{S}}_{[\mathrm{a}, \mathrm{b}]}(\phi, \Phi) \subseteq \overline{\mathrm{U}}_{[\mathrm{a}, \mathrm{b}]}(\phi, \Phi) .
$$

We have:

Theorem 2 Let $\mathrm{f}:[\mathrm{a}, \mathrm{b}] \rightarrow \mathbb{C}$ be a complex valued Lebesgue integrable function on the real interval $[\mathrm{a}, \mathrm{b}], \mathrm{g}$ be a strictly increasing function on $(\mathrm{a}, \mathrm{b})$, having a continuous derivative $\mathrm{g}^{\prime}$ on $(\mathrm{a}, \mathrm{b})$ and $\phi, \Phi \in \mathbb{C}, \phi \neq \Phi$ such that $\mathrm{f} \in \bar{\Delta}_{[\mathrm{a}, \mathrm{b}]}(\phi, \Phi)$.

(i) For any $\mathrm{x} \in(\mathrm{a}, \mathrm{b})$,

$$
\left|I_{a+, g}^{\alpha} f(x)+I_{b-, g}^{\alpha} f(x)-\frac{\phi+\Phi}{2 \Gamma(\alpha+1)}\left([g(x)-g(a)]^{\alpha}+[g(b)-g(x)]^{\alpha}\right)\right|
$$




$$
\leq \frac{1}{2}|\Phi-\phi| \frac{1}{\Gamma(\alpha+1)}\left[[g(x)-g(a)]^{\alpha}+[g(b)-g(x)]^{\alpha}\right]
$$

and

$$
\begin{aligned}
& \left|I_{x-, g}^{\alpha} f(a)+I_{x+, g}^{\alpha} f(b)-\frac{\phi+\Phi}{2 \Gamma(\alpha+1)}\left([g(x)-g(a)]^{\alpha}+[g(b)-g(x)]^{\alpha}\right)\right| \\
& \leq \frac{1}{2}|\Phi-\phi| \frac{1}{\Gamma(\alpha+1)}\left[[g(x)-g(a)]^{\alpha}+[g(b)-g(x)]^{\alpha}\right] .
\end{aligned}
$$

(ii) We have

$$
\begin{aligned}
& \left|\frac{I_{b-, g}^{\alpha} f(a)+I_{a+, g}^{\alpha} f(b)}{2}-\frac{1}{\Gamma(\alpha+1)}[g(b)-g(a)]^{\alpha} \frac{\phi+\Phi}{2}\right| \\
& \leq \frac{1}{2}|\Phi-\phi| \frac{1}{\Gamma(\alpha+1)}|\Phi-\phi|[g(b)-g(a)]^{\alpha} .
\end{aligned}
$$

Proof. Using the identity (1) for $\lambda=\mu=\frac{\phi+\Phi}{2}$, we have

$$
\begin{aligned}
I_{a+, g}^{\alpha} f(x)+ & I_{b-, g}^{\alpha} f(x) \\
& -\frac{1}{\Gamma(\alpha+1)}\left([g(x)-g(a)]^{\alpha}+[g(b)-g(x)]^{\alpha}\right) \frac{\phi+\Phi}{2} \\
= & \frac{1}{\Gamma(\alpha)}\left[\int_{a}^{x} \frac{g^{\prime}(t)\left[f(t)-\frac{\phi+\Phi}{2}\right] d t}{[g(x)-g(t)]^{1-\alpha}}+\int_{x}^{b} \frac{g^{\prime}(t)\left[f(t)-\frac{\phi+\Phi}{2}\right] d t}{[g(t)-g(x)]^{1-\alpha}}\right]
\end{aligned}
$$

for any $x \in(a, b)$.

Taking the modulus in (20), then we get

$$
\begin{aligned}
& \left|I_{a+, g}^{\alpha} f(x)+I_{b-, g}^{\alpha} f(x)-\frac{1}{\Gamma(\alpha+1)}\left([g(x)-g(a)]^{\alpha}+[g(b)-g(x)]^{\alpha}\right) \frac{\phi+\Phi}{2}\right| \\
& \leq \frac{1}{\Gamma(\alpha)}\left[\int_{a}^{x} \frac{g^{\prime}(t)\left|f(t)-\frac{\phi+\Phi}{2}\right| d t}{[g(x)-g(t)]^{1-\alpha}}+\int_{x}^{b} \frac{g^{\prime}(t)\left|f(t)-\frac{\phi+\Phi}{2}\right| d t}{[g(t)-g(x)]^{1-\alpha}}\right] \\
& \leq \frac{1}{2}|\Phi-\phi| \frac{1}{\Gamma(\alpha)}\left[\int_{a}^{x} \frac{g^{\prime}(t) d t}{[g(x)-g(t)]^{1-\alpha}}+\int_{x}^{b} \frac{g^{\prime}(t) d t}{[g(t)-g(x)]^{1-\alpha}}\right] \\
& \left.=\frac{1}{2}|\Phi-\phi| \frac{1}{\Gamma(\alpha+1)}[g(x)-g(a)]^{\alpha}+[g(b)-g(x)]^{\alpha}\right]
\end{aligned}
$$

for any $x \in(a, b)$, which proves (17). 
The inequality (18) follows in a similar manner from the identity (2).

The inequality (19) follows by (3) for $\lambda=\mu=\frac{\phi+\Phi}{2}$.

Corollary 5 With the assumptions of Theorem 2 we have

$$
\begin{aligned}
& \left|I_{a+, g}^{\alpha} f\left(M_{g}(a, b)\right)+I_{b-, g}^{\alpha} f\left(M_{g}(a, b)\right)-\frac{\phi+\Phi}{2^{\alpha} \Gamma(\alpha+1)}[g(b)-g(a)]^{\alpha}\right| \\
& \leq \frac{1}{2^{\alpha}}|\Phi-\phi| \frac{1}{\Gamma(\alpha+1)}[g(b)-g(a)]^{\alpha}
\end{aligned}
$$

and

$$
\begin{aligned}
& \left|I_{M_{g}(a, b)-, g}^{\alpha} f(a)+I_{M_{g}(a, b)+, g}^{\alpha} f(b)-\frac{\phi+\Phi}{2^{\alpha} \Gamma(\alpha+1)}[g(b)-g(a)]^{\alpha}\right| \\
& \leq \frac{1}{2^{\alpha}}|\Phi-\phi| \frac{1}{\Gamma(\alpha+1)}[g(b)-g(a)]^{\alpha} .
\end{aligned}
$$

Remark 4 If the function $\mathrm{f}:[\mathrm{a}, \mathrm{b}] \rightarrow \mathbb{R}$ is measurable and there exists the constants $\mathrm{m}, \mathrm{M}$ such that $\mathrm{m} \leq \mathrm{f}(\mathrm{t}) \leq \mathrm{M}$ for a.e. $\mathrm{t} \in[\mathrm{a}, \mathrm{b}]$, then for any $x \in(\mathrm{a}, \mathrm{b})$ we have by (17) and (18) that

$$
\begin{aligned}
& \left|I_{a+, g}^{\alpha} f(x)+I_{b-, g}^{\alpha} f(x)-\frac{m+M}{2 \Gamma(\alpha+1)}\left([g(x)-g(a)]^{\alpha}+[g(b)-g(x)]^{\alpha}\right)\right| \\
& \leq \frac{1}{2}(M-m) \frac{1}{\Gamma(\alpha+1)}\left[[g(x)-g(a)]^{\alpha}+[g(b)-g(x)]^{\alpha}\right]
\end{aligned}
$$

and

$$
\begin{aligned}
& \left|I_{x-, g}^{\alpha} f(a)+I_{x+, g}^{\alpha} f(b)-\frac{m+M}{2 \Gamma(\alpha+1)}\left([g(x)-g(a)]^{\alpha}+[g(b)-g(x)]^{\alpha}\right)\right| \\
& \leq \frac{1}{2}(M-m) \frac{1}{\Gamma(\alpha+1)}\left[[g(x)-g(a)]^{\alpha}+[g(b)-g(x)]^{\alpha}\right] .
\end{aligned}
$$

In particular,

$$
\begin{aligned}
& \left|I_{a+, g}^{\alpha} f\left(M_{g}(a, b)\right)+I_{b-, g}^{\alpha} f\left(M_{g}(a, b)\right)-\frac{m+M}{2^{\alpha} \Gamma(\alpha+1)}[g(b)-g(a)]^{\alpha}\right| \\
& \leq \frac{1}{2^{\alpha}}(M-m) \frac{1}{\Gamma(\alpha+1)}[g(b)-g(a)]^{\alpha}
\end{aligned}
$$

and

$$
\begin{aligned}
& \left|I_{M_{g}(a, b)-, g}^{\alpha} f(a)+I_{M_{g}(a, b)+, g}^{\alpha} f(b)-\frac{m+M}{2^{\alpha} \Gamma(\alpha+1)}[g(b)-g(a)]^{\alpha}\right| \\
& \leq \frac{1}{2^{\alpha}}(M-m) \frac{1}{\Gamma(\alpha+1)}[g(b)-g(a)]^{\alpha} .
\end{aligned}
$$




\section{Trapezoid inequalities for functions of bounded variation}

We have:

Theorem 3 Let $f:[a, b] \rightarrow \mathbb{C}$ be a complex valued function of bounded variation on the real interval $[\mathrm{a}, \mathrm{b}]$, and $\mathrm{g}$ be a strictly increasing function on $(\mathrm{a}, \mathrm{b})$, having a continuous derivative $\mathrm{g}^{\prime}$ on $(\mathrm{a}, \mathrm{b})$. Then we have the inequalities

$$
\begin{aligned}
& \left|I_{a+, g}^{\alpha} f(x)+I_{b-, g}^{\alpha} f(x)-\frac{[g(x)-g(a)]^{\alpha} f(a)+[g(b)-g(x)]^{\alpha} f(b)}{\Gamma(\alpha+1)}\right| \\
& \leq \frac{1}{\Gamma(\alpha)}\left[\int_{a}^{x} \frac{g^{\prime}(t) \bigvee_{a}^{t}(f) d t}{[g(x)-g(t)]^{1-\alpha}}+\int_{x}^{b} \frac{g^{\prime}(t) \bigvee_{t}^{b}(f) d t}{[g(t)-g(x)]^{1-\alpha}}\right] \\
& \leq \frac{1}{\Gamma(\alpha+1)}\left[(g(x)-g(a))^{\alpha} \bigvee_{a}^{x}(f)+(g(b)-g(x))^{\alpha} \bigvee_{x}^{b}(f)\right] \\
& \leq \frac{1}{\Gamma(\alpha+1)}\left\{\begin{array}{l}
\left((g(x)-g(a))^{\alpha p}+(g(b)-g(x))^{\alpha p}\right)^{1 / p}\left(\left(\bigvee_{a}^{x}(f)\right)^{q}+\left(\bigvee_{x}^{b}(f)\right)^{q}\right)^{1 / q} \\
w i t h p, q>1, \frac{1}{p}+\frac{1}{q}=1 ; \\
\left((g(x)-g(a))^{\alpha}+(g(b)-g(x))^{\alpha}\right)\left[\frac{1}{2} \bigvee_{a}^{b}(f)+\frac{1}{2}\left|\bigvee_{a}^{x}(f)-\bigvee_{x}^{b}(f)\right|\right]
\end{array}\right.
\end{aligned}
$$

and

$$
\begin{aligned}
& \left|I_{x-, g}^{\alpha} f(a)+I_{x+, g}^{\alpha} f(b)-\frac{[g(x)-g(a)]^{\alpha} f(a)+[g(b)-g(x)]^{\alpha} f(b)}{\Gamma(\alpha+1)}\right| \\
& \leq \frac{1}{\Gamma(\alpha)}\left[\int_{a}^{x} \frac{g^{\prime}(t) \bigvee_{a}^{t}(f) d t}{[g(t)-g(a)]^{1-\alpha}}+\int_{x}^{b} \frac{g^{\prime}(t) \bigvee_{t}^{b}(f) d t}{[g(b)-g(t)]^{1-\alpha}}\right] \\
& \leq \frac{1}{\Gamma(\alpha+1)}\left[\begin{array}{l}
(g(x)-g(a))^{\alpha} \bigvee_{a}^{x}(f)+(g(b)-g(x))^{\alpha} \bigvee_{x}^{b}(f)
\end{array}\right] \\
& \leq \frac{1}{\Gamma(\alpha+1)}\left\{\begin{array}{l}
{\left[\frac{1}{2}(g(b)-g(a))+\left|g(x)-\frac{g(a)+g(b)}{2}\right|\right]^{\alpha} \bigvee_{a}^{b}(f) ;} \\
\left.(g(x)-g(a))^{\alpha p}+(g(b)-g(x))^{\alpha p}\right)^{1 / p}\left(\left(\bigvee_{a}^{x}(f)\right)^{q}+\left(\bigvee_{x}^{b}(f)\right)^{q}\right)^{1 / q} \\
\left((g(x)-g(a))^{\alpha}+(g(b)-g(x))^{\alpha}\right)\left[\frac{1}{2} \bigvee_{a}^{b}(f)+\frac{1}{2}\left|\bigvee_{a}^{x}(f)-\bigvee_{x}^{b}(f)\right|\right]
\end{array}\right.
\end{aligned}
$$

for any $\mathrm{x} \in(\mathrm{a}, \mathrm{b})$ 
(ii) We also have

$$
\begin{aligned}
& \left|\frac{I_{b-, g}^{\alpha} f(a)+I_{a+, g}^{\alpha} f(b)}{2}-\frac{1}{\Gamma(\alpha+1)}[g(b)-g(a)]^{\alpha} \frac{f(b)+f(a)}{2}\right| \\
& \leq \frac{1}{2 \Gamma(\alpha)}\left[\int_{a}^{b} \frac{g^{\prime}(t) \bigvee_{t}^{b}(f) d t}{[g(b)-g(t)]^{1-\alpha}}+\int_{a}^{b} \frac{g^{\prime}(t) \bigvee_{a}^{t}(f) d t}{[g(t)-g(a)]^{1-\alpha}}\right] \\
& \leq \frac{1}{\Gamma(\alpha+1)}[g(b)-g(a)]^{\alpha} \bigvee_{a}^{b}(f) .
\end{aligned}
$$

Proof. Using the identity (13) and the properties of the modulus, we have

$$
\begin{aligned}
& \left|I_{a+, g}^{\alpha} f(x)+I_{b-, g}^{\alpha} f(x)-\frac{[g(x)-g(a)]^{\alpha} f(a)+[g(b)-g(x)]^{\alpha} f(b)}{\Gamma(\alpha+1)}\right| \\
& \leq \frac{1}{\Gamma(\alpha)}\left[\int_{a}^{x} \frac{g^{\prime}(t)|f(t)-f(a)| d t}{[g(x)-g(t)]^{1-\alpha}}+\int_{x}^{b} \frac{g^{\prime}(t)|f(t)-f(b)| d t}{[g(t)-g(x)]^{1-\alpha}}\right]=: B(x)
\end{aligned}
$$

for any $x \in(a, b)$.

Since $f$ is of bounded variation on $[a, b]$, then we have

$$
|f(t)-f(a)| \leq \bigvee_{a}^{t}(f) \leq \bigvee_{a}^{x}(f) \text { for } a \leq t \leq x
$$

and

$$
|f(t)-f(b)| \leq \bigvee_{t}^{b}(f) \leq \bigvee_{x}^{b}(f) \text { for } x \leq t \leq b
$$

Therefore

$$
\begin{aligned}
B(x) & \leq \frac{1}{\Gamma(\alpha)}\left[\int_{a}^{x} \frac{g^{\prime}(t) \bigvee_{a}^{t}(f) d t}{[g(x)-g(t)]^{1-\alpha}}+\int_{x}^{b} \frac{g^{\prime}(t) \bigvee_{t}^{b}(f) d t}{[g(t)-g(x)]^{1-\alpha}}\right] \\
& \leq \frac{1}{\Gamma(\alpha)}\left[\bigvee_{a}^{x}(f) \int_{a}^{x} \frac{g^{\prime}(t) d t}{[g(x)-g(t)]^{1-\alpha}}+\bigvee_{x}^{b}(f) \int_{x}^{b} \frac{g^{\prime}(t) d t}{[g(t)-g(x)]^{1-\alpha}}\right] \\
& =\frac{1}{\Gamma(\alpha)}\left[\frac{(g(x)-g(a))^{\alpha}}{\alpha} \bigvee_{a}^{x}(f)+\frac{(g(b)-g(x))^{\alpha}}{\alpha} \bigvee_{x}^{b}(f)\right] \\
& =\frac{1}{\Gamma(\alpha+1)}\left[(g(x)-g(a))^{\alpha} \bigvee_{a}^{x}(f)+(g(b)-g(x))^{\alpha} \bigvee_{x}^{b}(f)\right],
\end{aligned}
$$


which proves the first two inequalities in (21).

The last part of (21) is obvious by making use of the elementary Hölder type inequalities for positive real numbers $c, d, m, n \geq 0$

$$
m c+n d \leq\left\{\begin{array}{l}
\max \{m, n\}(c+d) \\
\left(m^{p}+n^{p}\right)^{1 / p}\left(c^{q}+d^{q}\right)^{1 / q} \text { with } p, q>1, \frac{1}{p}+\frac{1}{q}=1
\end{array}\right.
$$

The inequality (22) follows in a similar way by utilising the equality (14).

From the equality (15) we have

$$
\begin{aligned}
& \left|\frac{I_{b-, g}^{\alpha} f(a)+I_{a+, g}^{\alpha} f(b)}{2}-\frac{1}{\Gamma(\alpha+1)}[g(b)-g(a)]^{\alpha} \frac{f(b)+f(a)}{2}\right| \\
& \leq \frac{1}{2 \Gamma(\alpha)}\left[\int_{a}^{b} \frac{g^{\prime}(t)|f(t)-f(b)| d t}{[g(b)-g(t)]^{1-\alpha}}+\int_{a}^{b} \frac{g^{\prime}(t)|f(t)-f(a)| d t}{[g(t)-g(a)]^{1-\alpha}}\right] \\
& \leq \frac{1}{2 \Gamma(\alpha)}\left[\int_{a}^{b} \frac{g^{\prime}(t) \bigvee_{t}^{b}(f) d t}{[g(b)-g(t)]^{1-\alpha}}+\int_{a}^{b} \frac{g^{\prime}(t) \bigvee_{a}^{t}(f) d t}{[g(t)-g(a)]^{1-\alpha}}\right] \\
& \leq \frac{1}{2 \Gamma(\alpha)}\left[\bigvee_{a}^{b}(f) \int_{a}^{b} \frac{g^{\prime}(t) d t}{[g(b)-g(t)]^{1-\alpha}}+\bigvee_{a}^{b}(f) \int_{a}^{b} \frac{g^{\prime}(t) d t}{[g(t)-g(a)]^{1-\alpha}}\right] \\
& =\frac{1}{2 \Gamma(\alpha)}\left[\bigvee_{a}^{b}(f) \frac{[g(b)-g(a)]^{\alpha}}{\alpha}+\bigvee_{a}^{b}(f) \frac{[g(b)-g(a)]^{\alpha}}{\alpha}\right] \\
& =\frac{1}{\Gamma(\alpha+1)}[g(b)-g(a)]^{\alpha} \bigvee_{a}(f),
\end{aligned}
$$

which proves (23).

Corollary 6 With the assumptions of Theorem 3 we have

$$
\begin{aligned}
& \left|I_{a+, g}^{\alpha} f\left(M_{g}(a, b)\right)+I_{b-, g}^{\alpha} f\left(M_{g}(a, b)\right)-\frac{f(a)+f(b)}{2^{\alpha} \Gamma(\alpha+1)}[g(b)-g(a)]^{\alpha}\right| \\
& \leq \frac{1}{\Gamma(\alpha)}\left[\int_{a}^{M_{g}(a, b)} \frac{g^{\prime}(t) \bigvee_{a}^{t}(f) d t}{\left[g\left(M_{g}(a, b)\right)-g(t)\right]^{1-\alpha}}+\int_{M_{g}(a, b)}^{b} \frac{g^{\prime}(t) \bigvee_{t}^{b}(f) d t}{\left[g(t)-g\left(M_{g}(a, b)\right)\right]^{1-\alpha}}\right] \\
& \leq \frac{1}{2^{\alpha} \Gamma(\alpha+1)}(g(b)-g(a))^{\alpha} \bigvee_{a}^{b}(f)
\end{aligned}
$$


and

$$
\begin{aligned}
& \left|I_{M_{g}(a, b)-, g}^{\alpha} f(a)+I_{M_{g}(a, b)+, g}^{\alpha} f(b)-\frac{f(a)+f(b)}{2^{\alpha} \Gamma(\alpha+1)}[g(b)-g(a)]^{\alpha}\right| \\
& \leq \frac{1}{\Gamma(\alpha)}\left[\int_{a}^{M_{g}(a, b)} \frac{g^{\prime}(t) \bigvee_{a}^{t}(f) d t}{[g(t)-g(a)]^{1-\alpha}}+\int_{M_{g}(a, b)}^{b} \frac{g^{\prime}(t) \bigvee_{t}^{b}(f) d t}{[g(b)-g(t)]^{1-\alpha}}\right] \\
& \leq \frac{1}{2^{\alpha} \Gamma(\alpha+1)}(g(b)-g(a))^{\alpha} \bigvee_{a}^{b}(f) .
\end{aligned}
$$

\section{Inequalities for Hölder's continuous functions}

We say that the function $\mathrm{f}:[\mathrm{a}, \mathrm{b}] \rightarrow \mathbb{C}$ is $\mathrm{r}-\mathrm{H}$-Hölder continuous on $[\mathrm{a}, \mathrm{b}]$ with $r \in(0,1]$ and $\mathrm{H}>0$ if

$$
|f(t)-f(s)| \leq H|t-s|^{r}
$$

for any $t, s \in[a, b]$. If $r=1$ and $H=L$ we call the function L-Lipschitzian on $[a, b]$.

Theorem 4 Assume that $\mathrm{f}:[\mathrm{a}, \mathrm{b}] \rightarrow \mathbb{C}$ is $\mathrm{r}-\mathrm{H}$-Hölder continuous on $[\mathrm{a}, \mathrm{b}]$ with $\mathrm{r} \in(0,1]$ and $\mathrm{H}>0$, and $\mathrm{g}$ be a strictly increasing function on $(\mathrm{a}, \mathrm{b})$, having a continuous derivative $\mathrm{g}^{\prime}$ on $(\mathrm{a}, \mathrm{b})$. Then

$$
\begin{aligned}
& \left|I_{a+, g}^{\alpha} f(x)+I_{b-, g}^{\alpha} f(x)-\frac{[g(x)-g(a)]^{\alpha} f(a)+[g(b)-g(x)]^{\alpha} f(b)}{\Gamma(\alpha+1)}\right| \\
& \leq \frac{H}{\Gamma(\alpha)}\left[\int_{a}^{x} \frac{g^{\prime}(t)(t-a)^{r} d t}{[g(x)-g(t)]^{1-\alpha}}+\int_{x}^{b} \frac{g^{\prime}(t)(b-t)^{r} d t}{[g(t)-g(x)]^{1-\alpha}}\right] \\
& \leq \frac{H}{\Gamma(\alpha+1)}\left[(g(x)-g(a))^{\alpha}(x-a)^{r}+(g(b)-g(x))^{\alpha}(b-x)^{r}\right] \\
& \leq \frac{H}{\Gamma(\alpha+1)}\left\{\begin{array}{l}
{\left[\frac{1}{2}(g(b)-g(a))+\left|g(x)-\frac{g(a)+g(b)}{2}\right|\right]^{\alpha}\left[(x-a)^{r}+(b-x)^{r}\right] ;} \\
\left((g(x)-g(a))^{\alpha p}+(g(b)-g(x))^{\alpha p}\right)^{1 / p}\left((x-a)^{r q}+(b-x)^{r q}\right)^{1 / q} \\
\left((g(x)-g(a))^{\alpha}+(g(b)-g(x))^{\alpha}\right)\left[\frac{1}{2}(b-a)+\left|x-\frac{a+b}{2}\right|\right]^{r}
\end{array}\right.
\end{aligned}
$$


and

$$
\begin{aligned}
& \left|I_{x-, g}^{\alpha} f(a)+I_{x+, g}^{\alpha} f(b)-\frac{[g(x)-g(a)]^{\alpha} f(a)+[g(b)-g(x)]^{\alpha} f(b)}{\Gamma(\alpha+1)}\right| \\
& \leq \frac{H}{\Gamma(\alpha)}\left[\int_{a}^{x} \frac{g^{\prime}(t)(t-a)^{r} d t}{[g(t)-g(a)]^{1-\alpha}}+\int_{x}^{b} \frac{g^{\prime}(t)(b-t)^{r} d t}{[g(b)-g(t)]^{1-\alpha}}\right] \\
& \leq \frac{H}{\Gamma(\alpha+1)}\left[(g(x)-g(a))^{\alpha}(x-a)^{r}+(g(b)-g(x))^{\alpha}(b-x)^{r}\right] \\
& \leq \frac{H}{\Gamma(\alpha+1)}\left\{\begin{array}{l}
{\left[\frac{1}{2}(g(b)-g(a))+\left|g(x)-\frac{g(a)+g(b)}{2}\right|\right]^{\alpha}\left[(x-a)^{r}+(b-x)^{r}\right] ;} \\
\left((g(x)-g(a))^{\alpha p}+(g(b)-g(x))^{\alpha p}\right)^{1 / p}\left((x-a)^{r q}+(b-x)^{r q}\right)^{1 / q} \\
\left((g(x)-g(a))^{\alpha}+(g(b)-g(x))^{\alpha}\right)\left[\frac{1}{2}(b-a)+\left|x-\frac{a+b}{2}\right|\right]^{r}
\end{array}\right.
\end{aligned}
$$

for any $\mathrm{x} \in(\mathrm{a}, \mathrm{b})$

(ii) We also have

$$
\begin{aligned}
& \left|\frac{I_{b-, g}^{\alpha} f(a)+I_{a+, g}^{\alpha} f(b)}{2}-\frac{1}{\Gamma(\alpha+1)}[g(b)-g(a)]^{\alpha} \frac{f(b)+f(a)}{2}\right| \\
& \leq \frac{H}{2 \Gamma(\alpha)}\left[\int_{a}^{b} \frac{g^{\prime}(t)(b-t)^{r} d t}{[g(b)-g(t)]^{1-\alpha}}+\int_{a}^{b} \frac{g^{\prime}(t)(t-a)^{r} d t}{[g(t)-g(a)]^{1-\alpha}}\right] \\
& \leq \frac{H}{\Gamma(\alpha+1)}[g(b)-g(a)]^{\alpha}(b-a)^{r} .
\end{aligned}
$$

Proof. Using the identity (13) and the properties of the modulus, we have

$$
\begin{aligned}
& \left|I_{a+, g}^{\alpha} f(x)+I_{b-, g}^{\alpha} f(x)-\frac{[g(x)-g(a)]^{\alpha} f(a)+[g(b)-g(x)]^{\alpha} f(b)}{\Gamma(\alpha+1)}\right| \\
& \leq \frac{1}{\Gamma(\alpha)}\left[\int_{a}^{x} \frac{g^{\prime}(t)|f(t)-f(a)| d t}{[g(x)-g(t)]^{1-\alpha}}+\int_{x}^{b} \frac{g^{\prime}(t)|f(t)-f(b)| d t}{[g(t)-g(x)]^{1-\alpha}}\right]=: C(x)
\end{aligned}
$$

for any $x \in(a, b)$.

Since $f:[a, b] \rightarrow \mathbb{C}$ is $r-H$-Hölder continuous on $[a, b]$ with $r \in(0,1]$ and $\mathrm{H}>0$, hence

$$
C(x) \leq \frac{H}{\Gamma(\alpha)}\left[\int_{a}^{x} \frac{g^{\prime}(t)(t-a)^{r} d t}{[g(x)-g(t)]^{1-\alpha}}+\int_{x}^{b} \frac{g^{\prime}(t)(b-t)^{r} d t}{[g(t)-g(x)]^{1-\alpha}}\right]
$$




$$
\begin{aligned}
& \leq \frac{H}{\Gamma(\alpha)}\left[(x-a)^{r} \int_{a}^{x} \frac{g^{\prime}(t) d t}{[g(x)-g(t)]^{1-\alpha}}+(b-x)^{r} \int_{x}^{b} \frac{g^{\prime}(t) d t}{[g(t)-g(x)]^{1-\alpha}}\right] \\
& =\frac{H}{\Gamma(\alpha)}\left[(x-a)^{r} \frac{(g(x)-g(a))^{\alpha}}{\alpha}+(b-x)^{r} \frac{(g(b)-g(x))^{\alpha}}{\alpha}\right] \\
& =\frac{H}{\Gamma(\alpha+1)}\left[(x-a)^{r}(g(x)-g(a))^{\alpha}+(b-x)^{r}(g(b)-g(x))^{\alpha}\right],
\end{aligned}
$$

for any $x \in(a, b)$, which proves the first two inequalities in (25). The rest is obvious.

The inequality (26) follows in a similar way by utilising the equality (14).

The inequality (27) follows by utilising the equality (15).

Corollary 7 With the assumptions of Theorem 4 we have

$$
\begin{aligned}
& \left|I_{a+, g}^{\alpha} f\left(M_{g}(a, b)\right)+I_{b-, g}^{\alpha} f\left(M_{g}(a, b)\right)-\frac{f(a)+f(b)}{2^{\alpha} \Gamma(\alpha+1)}[g(b)-g(a)]^{\alpha}\right| \\
& \leq \frac{H}{\Gamma(\alpha)}\left[\int_{a}^{M_{g}(a, b)} \frac{g^{\prime}(t)(t-a)^{r} d t}{\left[g\left(M_{g}(a, b)\right)-g(t)\right]^{1-\alpha}}+\int_{M_{g}(a, b)}^{b} \frac{g^{\prime}(t)(b-t)^{r} d t}{\left[g(t)-g\left(M_{g}(a, b)\right)\right]^{1-\alpha}}\right] \\
& \leq \frac{H}{2^{\alpha} \Gamma(\alpha+1)}(g(b)-g(a))^{\alpha}\left[\left(M_{g}(a, b)-a\right)^{r}+\left(b-M_{g}(a, b)\right)^{r}\right]
\end{aligned}
$$

and

$$
\begin{aligned}
& \left|I_{M_{g}(a, b)-, g}^{\alpha} f(a)+I_{M_{g}(a, b)+, g}^{\alpha} f(b)-\frac{f(a)+f(b)}{2^{\alpha} \Gamma(\alpha+1)}[g(b)-g(a)]^{\alpha}\right| \\
& \leq \frac{H}{\Gamma(\alpha)}\left[\int_{a}^{M_{g}(a, b)} \frac{g^{\prime}(t)(t-a)^{r} d t}{[g(t)-g(a)]^{1-\alpha}}+\int_{M_{g}(a, b)}^{b} \frac{g^{\prime}(t)(b-t)^{r} d t}{[g(b)-g(t)]^{1-\alpha}}\right] \\
& \leq \frac{H}{2^{\alpha} \Gamma(\alpha+1)}(g(b)-g(a))^{\alpha}\left[\left(M_{g}(a, b)-a\right)^{r}+\left(b-M_{g}(a, b)\right)^{r}\right] .
\end{aligned}
$$

\section{Applications for Hadamard fractional integrals}

If we take $g(t)=\ln t$ and $0 \leq a<x \leq b$, then by Theorem 3 for Hadamard fractional integrals $H_{a+}^{\alpha}$ and $H_{b-}^{\alpha}$ we have for $f:[a, b] \rightarrow \mathbb{C}$, a function of bounded variation on $[a, b]$ that

$$
\left|H_{a+}^{\alpha} f(x)+H_{b-}^{\alpha} f(x)-\frac{\left[\ln \left(\frac{x}{a}\right)\right]^{\alpha} f(a)+\left[\ln \left(\frac{b}{x}\right)\right]^{\alpha} f(b)}{\Gamma(\alpha+1)}\right|
$$


48

S. S. Dragomir

$$
\begin{aligned}
& \leq \frac{1}{\Gamma(\alpha)}\left[\int_{a}^{x} \frac{\left[\ln \left(\frac{x}{t}\right)\right]^{\alpha-1} \bigvee_{a}^{t}(f) d t}{t}+\int_{x}^{b} \frac{\left[\ln \left(\frac{t}{x}\right)\right]^{\alpha-1} \bigvee_{t}^{b}(f) d t}{t}\right] \\
& \leq \frac{1}{\Gamma(\alpha+1)}\left[\left[\ln \left(\frac{x}{a}\right)\right]^{\alpha} \bigvee_{a}^{x}(f)+\left[\ln \left(\frac{b}{x}\right)\right]_{x}^{\alpha} \bigvee^{b}(f)\right] \\
& \leq \frac{1}{\Gamma(\alpha+1)}\left\{\begin{array}{l}
{\left[\frac{1}{2} \ln \left(\frac{b}{a}\right)+\left|\ln \left(\frac{x}{G(a, b)}\right)\right|\right]_{a}^{\alpha} \bigvee_{a}^{b}(f) ;} \\
\left(\left(\ln \left(\frac{x}{a}\right)\right)^{\alpha p}+\left(\ln \left(\frac{b}{x}\right)\right)^{\alpha p}\right)^{1 / p}\left(\left(\bigvee_{a}^{x}(f)\right)^{q}+\left(\bigvee_{x}^{b}(f)\right)^{q}\right)^{1 / q} \\
\left(\left(\ln \left(\frac{x}{a}\right)\right)^{\alpha}+\left(\ln \left(\frac{b}{x}\right)\right)^{\alpha}\right)\left[\frac{1}{2} \bigvee_{a}^{b}(f)+\frac{1}{2}\left|\bigvee_{a}^{x}(f)-\bigvee_{x}^{b}(f)\right|\right]
\end{array}\right.
\end{aligned}
$$

and

$$
\begin{aligned}
& \left|H_{x-}^{\alpha} f(a)+H_{x+}^{\alpha} f(b)-\frac{\left[\ln \left(\frac{x}{a}\right)\right]^{\alpha} f(a)+\left[\ln \left(\frac{b}{x}\right)\right]^{\alpha} f(b)}{\Gamma(\alpha+1)}\right| \\
& \leq \frac{1}{\Gamma(\alpha)}\left[\int_{a}^{x} \frac{\left[\ln \left(\frac{t}{a}\right)\right]^{\alpha-1} \bigvee_{a}^{t}(f) d t}{t}+\int_{x}^{b} \frac{\left[\ln \left(\frac{b}{t}\right)\right]^{\alpha-1} \bigvee_{t}^{b}(f) d t}{t}\right] \\
& \leq \frac{1}{\Gamma(\alpha+1)}\left[\left(\ln \left(\frac{x}{a}\right)\right)^{\alpha} \bigvee_{a}^{x}(f)+\left(\ln \left(\frac{b}{x}\right)\right)^{\alpha} \bigvee_{x}^{b}(f)\right] \\
& \leq \frac{1}{\Gamma(\alpha+1)}\left\{\begin{array}{l}
{\left[\frac{1}{2} \ln \left(\frac{b}{a}\right)+\left|\ln \left(\frac{x}{G(a, b)}\right)\right|\right]^{\alpha} \bigvee_{a}^{b}(f) ;} \\
\left(\left(\ln \left(\frac{x}{a}\right)\right)^{\alpha p}+\left(\ln \left(\frac{b}{x}\right)\right)^{\alpha p}\right)^{1 / p}\left(\left(\bigvee_{a}^{x}(f)\right)^{q}+\left(\bigvee_{x}^{b}(f)\right)^{q}\right)^{1 / q} \\
\text { with } p, q>1, \frac{1}{p}+\frac{1}{q}=1 ; \\
\left(\left(\ln \left(\frac{x}{a}\right)\right)^{\alpha}+\left(\ln \left(\frac{b}{x}\right)\right)^{\alpha}\right)\left[\frac{1}{2} \bigvee_{a}^{b}(f)+\frac{1}{2}\left|\bigvee_{a}^{x}(f)-\bigvee_{x}^{b}(f)\right|\right]
\end{array}\right.
\end{aligned}
$$

for any $x \in(a, b)$

We also have

$$
\begin{aligned}
& \left|\frac{H_{b-}^{\alpha} f(a)+H_{a+}^{\alpha} f(b)}{2}-\frac{1}{\Gamma(\alpha+1)}\left[\ln \left(\frac{b}{a}\right)\right]^{\alpha} \frac{f(b)+f(a)}{2}\right| \\
& \leq \frac{1}{2 \Gamma(\alpha)}\left[\int_{a}^{b} \frac{\left[\ln \left(\frac{b}{t}\right)\right]^{\alpha-1} \bigvee_{t}^{b}(f) d t}{t}+\int_{a}^{b} \frac{\left[\ln \left(\frac{t}{a}\right)\right]^{\alpha-1} g^{\prime}(t) \bigvee_{a}^{t}(f) d t}{t}\right] \\
& \leq \frac{1}{\Gamma(\alpha+1)}\left[\ln \left(\frac{b}{a}\right)\right]_{a}^{\alpha} b(f) .
\end{aligned}
$$


If we take in (28) and (29) $x=G(a, b)$, then we get

$$
\begin{aligned}
& \left|H_{a+}^{\alpha} f(G(a, b))+H_{b-}^{\alpha} f(G(a, b))-\frac{f(a)+f(b)}{2^{\alpha} \Gamma(\alpha+1)}\left[\ln \left(\frac{b}{a}\right)\right]^{\alpha}\right| \\
& \leq \frac{1}{\Gamma(\alpha)}\left[\int_{a}^{G(a, b)} \frac{\left[\ln \left(\frac{G(a, b)}{t}\right)\right]^{\alpha-1} V_{a}^{t}(f) d t}{t}+\int_{G(a, b)}^{b} \frac{\left[\ln \left(\frac{t}{G(a, b)}\right)\right]^{\alpha-1} \bigvee_{t}^{b}(f) d t}{t}\right] \\
& \leq \frac{1}{2^{\alpha} \Gamma(\alpha+1)}\left[\ln \left(\frac{b}{a}\right)\right]^{\alpha} \bigvee_{a}^{b}(f)
\end{aligned}
$$

and

$$
\begin{aligned}
& \left|H_{G(a, b)-}^{\alpha} f(a)+H_{G(a, b)+}^{\alpha} f(b)-\frac{f(a)+f(b)}{2^{\alpha} \Gamma(\alpha+1)}\left[\ln \left(\frac{b}{a}\right)\right]^{\alpha}\right| \\
& \leq \frac{1}{\Gamma(\alpha)}\left[\int_{a}^{G(a, b)} \frac{\left[\ln \left(\frac{t}{a}\right)\right]^{\alpha-1} \bigvee_{a}^{t}(f) d t}{t}+\int_{G(a, b)}^{b} \frac{\left[\ln \left(\frac{b}{t}\right)\right]^{\alpha-1} \bigvee_{t}^{b}(f) d t}{t}\right] \\
& \leq \frac{1}{2^{\alpha} \Gamma(\alpha+1)}\left[\ln \left(\frac{b}{a}\right)\right]^{\alpha} \bigvee_{a}^{b}(f) .
\end{aligned}
$$

Assume that $f:[a, b] \rightarrow \mathbb{C}$ is $r-H$-Hölder continuous on $[a, b]$ with $r \in(0,1]$ and $H>0$. If we take $g(t)=\ln t$ and $0 \leq a<x \leq b$ in Theorem 4, then we get

$$
\begin{aligned}
& \left|H_{a+}^{\alpha} f(x)+H_{b-}^{\alpha} f(x)-\frac{\left[\ln \left(\frac{x}{a}\right)\right]^{\alpha} f(a)+\left[\ln \left(\frac{b}{x}\right)\right]^{\alpha} f(b)}{\Gamma(\alpha+1)}\right| \\
& \leq \frac{H}{\Gamma(\alpha)}\left[\int_{a}^{x} \frac{\left[\ln \left(\frac{x}{t}\right)\right]^{\alpha-1}(t-a)^{r} d t}{t}+\int_{x}^{b} \frac{\left[\ln \left(\frac{t}{x}\right)\right]^{\alpha-1}(b-t)^{r} d t}{t}\right] \\
& \leq \frac{H}{\Gamma(\alpha+1)}\left[\left[\ln \left(\frac{x}{a}\right)\right]^{\alpha}(x-a)^{r}+\left[\ln \left(\frac{b}{x}\right)\right]^{\alpha}(b-x)^{r}\right] \\
& \leq \frac{H}{\Gamma(\alpha+1)}\left\{\begin{array}{l}
{\left[\frac{1}{2} \ln \left(\frac{b}{a}\right)+\left|\ln \left(\frac{x}{G(a, b)}\right)\right|\right]^{\alpha} \bigvee_{a}^{b}(f) ;} \\
\left(\left(\ln \left(\frac{x}{a}\right)\right)^{\alpha p}+\left(\ln \left(\frac{b}{x}\right)\right)^{\alpha p}\right)^{1 / p}\left(\left(\bigvee_{a}^{x}(f)\right)^{q}+\left(\bigvee_{x}^{b}(f)\right)^{q}\right)^{1 / q} \\
\left(\left(\ln \left(\frac{x}{a}\right)\right)^{\alpha}+\left(\ln \left(\frac{b}{x}\right)\right)^{\alpha}\right)\left[\frac{1}{2} \bigvee_{a}^{b}(f)+\frac{1}{2}\left|\bigvee_{a}^{x}(f)-\bigvee_{x}^{b}(f)\right|\right]
\end{array}\right.
\end{aligned}
$$


and

$$
\begin{aligned}
& \left|H_{x-}^{\alpha} f(a)+H_{x+}^{\alpha} f(b)-\frac{\left[\ln \left(\frac{x}{a}\right)\right]^{\alpha} f(a)+\left[\ln \left(\frac{b}{x}\right)\right]^{\alpha} f(b)}{\Gamma(\alpha+1)}\right| \\
& \leq \frac{H}{\Gamma(\alpha)}\left[\int_{a}^{x} \frac{\left[\ln \left(\frac{t}{a}\right)\right]^{\alpha-1}(t-a)^{r} d t}{t}+\int_{x}^{b} \frac{\left[\ln \left(\frac{b}{t}\right)\right]^{\alpha-1}(b-t)^{r} d t}{t}\right] \\
& \leq \frac{H}{\Gamma(\alpha+1)}\left[\left[\ln \left(\frac{x}{a}\right)\right]^{\alpha}(x-a)^{r}+\left[\ln \left(\frac{b}{x}\right)\right]^{\alpha}(b-x)^{r}\right] \\
& \leq \frac{H}{\Gamma(\alpha+1)}\left\{\begin{array}{l}
{\left[\frac{1}{2} \ln \left(\frac{b}{a}\right)+\left|\ln \left(\frac{x}{G(a, b)}\right)\right|\right]^{\alpha} \bigvee_{a}^{b}(f) ;} \\
\left(\left(\ln \left(\frac{x}{a}\right)\right)^{\alpha p}+\left(\ln \left(\frac{b}{x}\right)\right)^{\alpha p}\right)^{1 / p}\left(\left(\bigvee_{a}^{x}(f)\right)^{q}+\left(\bigvee_{x}^{b}(f)\right)^{q}\right)^{1 / q} \\
\left(\left(\ln \left(\frac{x}{a}\right)\right)^{\alpha}+\left(\ln \left(\frac{b}{x}\right)\right)^{\alpha}\right)\left[\frac{1}{2} \bigvee_{a}^{b}(f)+\frac{1}{2}\left|\bigvee_{a}^{x}(f)-\bigvee_{x}^{b}(f)\right|\right]
\end{array}\right.
\end{aligned}
$$

for any $x \in(a, b)$.

We also have

$$
\begin{aligned}
& \left|\frac{H_{b-}^{\alpha} f(a)+H_{a+}^{\alpha} f(b)}{2}-\frac{1}{\Gamma(\alpha+1)}\left[\ln \left(\frac{b}{a}\right)\right]^{\alpha} \frac{f(b)+f(a)}{2}\right| \\
& \leq \frac{H}{2 \Gamma(\alpha)}\left[\int_{a}^{b} \frac{\left[\ln \left(\frac{b}{t}\right)\right]^{\alpha-1}(b-t)^{r} d t}{t}+\int_{a}^{b} \frac{\left[\ln \left(\frac{t}{a}\right)\right]^{\alpha-1}(t-a)^{r} d t}{t}\right] \\
& \leq \frac{H}{\Gamma(\alpha+1)}(b-a)^{r}\left[\ln \left(\frac{b}{a}\right)\right]^{\alpha} .
\end{aligned}
$$

If we take in (30) and (31) $x=G(a, b)$, then we get

$$
\begin{aligned}
& \left|H_{a+}^{\alpha} f(G(a, b))+H_{b-}^{\alpha} f(G(a, b))-\frac{f(a)+f(b)}{2^{\alpha} \Gamma(\alpha+1)}\left[\ln \left(\frac{b}{a}\right)\right]^{\alpha}\right| \\
& \leq \frac{H}{\Gamma(\alpha)}\left[\int_{a}^{G(a, b)} \frac{\left[\ln \left(\frac{G(a, b)}{t}\right)\right]^{\alpha-1}(t-a)^{r} d t}{t}+\int_{G(a, b)}^{b} \frac{\left[\ln \left(\frac{t}{G(a, b)}\right)\right]^{\alpha-1}(b-t)^{r} d t}{t}\right] \\
& \leq \frac{1}{2^{\alpha} \Gamma(\alpha+1)}\left[\ln \left(\frac{b}{a}\right)\right]^{\alpha}(b-a)^{r}
\end{aligned}
$$

and

$$
\left|H_{G(a, b)-}^{\alpha} f(a)+H_{G(a, b)+}^{\alpha} f(b)-\frac{f(a)+f(b)}{2^{\alpha} \Gamma(\alpha+1)}\left[\ln \left(\frac{b}{a}\right)\right]^{\alpha}\right|
$$




$$
\begin{aligned}
& \leq \frac{1}{\Gamma(\alpha)}\left[\int_{a}^{G(a, b)} \frac{\left[\ln \left(\frac{t}{a}\right)\right]^{\alpha-1}(t-a)^{r} d t}{t}+\int_{G(a, b)}^{b} \frac{\left[\ln \left(\frac{b}{t}\right)\right]^{\alpha-1}(b-t)^{r} d t}{t}\right] \\
& \leq \frac{1}{2^{\alpha} \Gamma(\alpha+1)}\left[\ln \left(\frac{b}{a}\right)\right]^{\alpha}(b-a)^{r} .
\end{aligned}
$$

\section{Acknowledgement}

The author would like to thank the anonymous referee for valuable suggestions that have been implemented in the final version of the paper.

\section{References}

[1] A. Aglić Aljinović, Montgomery identity and Ostrowski type inequalities for Riemann-Liouville fractional integral, J. Math., 2014, Art. ID 503195, $6 \mathrm{pp}$.

[2] A. O. Akdemir, Inequalities of Ostrowski's type for $m$ - and $(\alpha, m)$ logarithmically convex functions via Riemann-Liouville fractional integrals, J. Comput. Anal. Appl., 16 (2014), no. 2, 375-383

[3] G. A. Anastassiou, Fractional representation formulae under initial conditions and fractional Ostrowski type inequalities, Demonstr. Math., 48 (2015), no. $3,357-378$

[4] G. A. Anastassiou, The reduction method in fractional calculus and fractional Ostrowski type inequalities, Indian J. Math., 56 (2014), no. 3, 333357.

[5] H. Budak, M. Z. Sarikaya, E. Set, Generalized Ostrowski type inequalities for functions whose local fractional derivatives are generalized s-convex in the second sense, J. Appl. Math. Comput. Mech., 15 (2016), no. 4, 11-21.

[6] P. Cerone and S. S. Dragomir, Midpoint-type rules from an inequalities point of view, Handbook of analytic-computational methods in applied mathematics, 135-200, Chapman \& Hall/CRC, Boca Raton, FL, 2000.

[7] S. S. Dragomir, The Ostrowski's integral inequality for Lipschitzian mappings and applications, Comput. Math. Appl., 38 (1999), no. 11-12, 33-37.

[8] S. S. Dragomir, The Ostrowski integral inequality for mappings of bounded variation, Bull. Austral. Math. Soc. 60 (1999), No. 3, 495-508. 
[9] S. S. Dragomir, On the midpoint quadrature formula for mappings with bounded variation and applications, Kragujevac J. Math., 22 (2000), 1319.

[10] S. S. Dragomir, On the Ostrowski's integral inequality for mappings with bounded variation and applications, Math. Ineq. Appl.,4 (2001), No. 1, 59-66. Preprint: RGMIA Res. Rep. Coll. 2 (1999), Art. 7, [Online: http://rgmia.org/papers/v2n1/v2n1-7.pdf]

[11] S. S. Dragomir, Refinements of the Ostrowski inequality in terms of the cumulative variation and applications, Analysis (Berlin) 34 (2014), No. 2, 223-240. Preprint: RGMIA Res. Rep. Coll., 16 (2013), Art. 29 [Online:http://rgmia.org/papers/v16/v16a29.pdf] .

[12] S. S. Dragomir, Ostrowski type inequalities for Lebesgue integral: a survey of recent results, Australian J. Math. Anal. Appl., Volume 14, Issue 1, Article 1, pp. 1-287, 2017. [Online http://ajmaa.org/cgi-bin/paper.pl?string=v14n1/V14I1P1.tex] .

[13] S. S. Dragomir, Ostrowski type inequalities for Riemann-Liouville fractional integrals of bounded variation, Hölder and Lipschitzian functions, Preprint RGMIA Res. Rep. Coll., 20 (2017), Art. 48. [Online http://rgmia.org/papers/v20/v20a48.pdf] .

[14] S. S. Dragomir, Ostrowski type inequalities for generalized RiemannLiouville fractional integrals of functions with bounded variation, Preprint RGMIA Res. Rep. Coll., 20 (2017), Art 58. [Online http://rgmia.org/papers/v20/v20a58.pdf] .

[15] S. S. Dragomir, M. S. Moslehian and Y. J. Cho, Some reverses of the Cauchy-Schwarz inequality for complex functions of self-adjoint operators in Hilbert spaces,Math. Inequal. Appl., 17 (2014), no. 4, 1365-1373.

[16] A. Guezane-Lakoud and F. Aissaoui, New fractional inequalities of Ostrowski type, Transylv. J. Math. Mech., 5 (2013), no. 2, 103-106

[17] A. Kashuri and R. Liko, Ostrowski type fractional integral inequalities for generalized (s, m, $\varphi$ )-preinvex functions, Aust. J. Math. Anal. Appl., 13 (2016), no. 1, Art. 16, 11 pp.

[18] A. Kilbas, A; H. M. Srivastava and J. J. Trujillo, Theory and Applications of Fractional Differential Equations, North-Holland Mathematics Studies, 
204. Elsevier Science B.V., Amsterdam, 2006. xvi+523 pp. ISBN: 978-0444-51832-3; 0-444-51832-0

[19] M. A. Noor, K. I.Noor and S. Iftikhar, Fractional Ostrowski inequalities for harmonic h-preinvex functions, Facta Univ. Ser. Math. Inform., 31 (2016), no. 2, 417-445

[20] M. Z. Sarikaya and H. Filiz, Note on the Ostrowski type inequalities for fractional integrals, Vietnam J. Math., 42 (2014), no. 2, 187-190

[21] M. Z. Sarikaya and H. Budak, Generalized Ostrowski type inequalities for local fractional integrals, Proc. Amer. Math. Soc., 145 (2017), no. 4, $1527-1538$.

[22] E. Set, New inequalities of Ostrowski type for mappings whose derivatives are s-convex in the second sense via fractional integrals, Comput. Math. Appl., 63 (2012), no. 7, 1147-1154.

[23] M. Tunç, On new inequalities for $h$-convex functions via RiemannLiouville fractional integration, Filomat, 27:4 (2013), 559-565.

[24] M. Tunç, Ostrowski type inequalities for $m$ - and $(\alpha, m)$-geometrically convex functions via Riemann-Louville fractional integrals, Afr. Mat., 27 (2016), no. 5-6, 841-850.

[25] H. Yildirim and Z. Kirtay, Ostrowski inequality for generalized fractional integral and related inequalities, Malaya J. Mat., 2 (3) (2014), 322-329.

[26] C. Yildiz, E, Özdemir and Z. S. Muhamet, New generalizations of Ostrowski-like type inequalities for fractional integrals, Kyungpook Math. J. 56 (2016), no. 1, 161-172.

[27] H. Yue, Ostrowski inequality for fractional integrals and related fractional inequalities, Transylv. J. Math. Mech., 5 (2013), no. 1, 85-89. 